\title{
Establishment of cancer/testis antigen profiling based on clinicopathological characteristics in resected pathological stage III non-small cell
} lung cancer

This article was published in the following Dove Press journal:

Cancer Management and Research

\author{
Shi Jin',* \\ Shoubo Cao ${ }^{1,2, *}$ \\ Aleksei Grigorev 3 ,* \\ Jianhua $\mathrm{Li}^{4}$ \\ Qingwei Meng' \\ Chunyan Wang ${ }^{1,2}$ \\ Meiyan Feng 5 \\ Jing $\mathrm{Hu}^{\prime}$ \\ Feng Jiang ${ }^{3}$ \\ Yan Yu'
}

'Department of Medical Oncology, Harbin Medical University Cancer Hospital, Harbin, People's Republic of China; ${ }^{2}$ Department of Medical Oncology, Linyi People's Hospital, Linyi City, People's Republic of

China; ${ }^{3}$ School of Computer Science and Technology, Harbin Institute of Technology, Harbin, People's Republic of China; ${ }^{4}$ Department of Neurosurgery, The Fourth Affiliated Hospital of Harbin Medical University, Harbin, People's Republic of China ${ }^{5}$ Department of Pathology, Harbin Medical University Cancer Hospital, Harbin, People's Republic of China

*These authors contributed equally to this work

Correspondence: Yan Yu

Department of Medical Oncology, Harbin Medical University Cancer Hospital,

No. I50 Haping Road, Harbin I5008I,

People's Republic of China

Tel/Fax +86 45I 86298727

Email yuyan@ems.hrbmu.edu.cn

Feng Jiang

School of Computer Science and

Technology, Harbin Institute of

Technology, No. 92 West Da Zhi Street, Harbin I5000I, People's Republic of China Email fjiang@hit.edu.cn
Background: Cancer/testis antigen (CTA) expression was found to be highly heterogeneous in previous studies. We aimed to establish a precision CTA profiling in resected stage III nonsmall cell lung cancer (NSCLC) and demonstrate the best CTA combination covering the widest range of NSCLC cases.

Materials and methods: The expression of 10 CTAs was evaluated in 200 resected stage III NSCLC tissue specimens at protein level. Hierarchical clustering and python programming language analyses was used to demonstrate CTA expression and coverage.

Results: The most commonly expressed CTAs for total cases were MAGEA1 $(60.0 \%)$, MAGEA10 (50.0\%), and KK-LC-1 (47.5\%). CTA expression was histology dependent, and concurrent expression was common. The best 2,3 , and 4 CTA combination covered $72.0 \%$, $76.5 \%$, and $79.5 \%$ of total cases, respectively. Stratified analysis based on variable clinicopathological characteristics achieved the maximum coverage of $92.3 \%$ with only 2 CTA combination in patients with features of male sex, positive smoking history, and adenocarcinoma, compared with a $85.0 \%$ coverage when 10 CTAs were assessed. Selected CTA expression was correlated with prognosis based on subgroup analysis. No significant difference was found between CTA expression and epidermal growth factor receptor mutant status.

Conclusion: We established an individualized CTA profiling in resected stage III NSCLC based on 10 CTA expression. With the help of computer programming language, the goal of the maximum CTA expression coverage was reached by using the least CTA combination based on sex, smoking history, and histology. These results were significant for the further study of CTA-specific T-cell immunotherapy.

Keywords: lung cancer, cancer/testis antigens, immunotherapy, prognosis, profiling

\section{Introduction}

Lung cancer remains the most frequent newly diagnosed and first leading cause of cancer-related deaths worldwide. ${ }^{1-3}$ In more developed countries, it has even overpassed breast cancer as the leading cause of cancer death in females. In People's Republic of China, lung cancer incidence has been increasing and the new diagnosed lung cancer cases in year 2011 increased by $34.8 \%$ compared with 2005 data. $^{2}$ In addition to high incidence and mortality, lung cancer prognosis remains unsatisfied, with a 5-year survival of $<10 \% .^{2}$ Treatment strategies with high efficiency and safety profiles are urgently needed.

Antigen-specific T-cell immunotherapy has become an active area of cancer immunotherapy in recent years. Previous studies have demonstrated that antigen-specific 
T-cell immunotherapy can induce a phenotypic and functional conversion of $\mathrm{T}$ cells into a central memory type, resulting in $50 \%-80 \%$ disease stabilization in refractory and progressive metastatic melanoma patients. Continuous complete response was even seen in one patient for over 3 years. ${ }^{4,5}$ Another study involving relapsed or high-risk leukemia showed a response rate of over $60 \%$ induced by long-lived memory T cells. ${ }^{6}$ All studies give us great encouragement for the long-term efficient anti-tumor effect and safety profiles of antigen-specific T-cell immunotherapy.$^{46}$ However, not all malignancies have potential targets with high immunogenicity. ${ }^{7}$ The lack of effective targets is the most important element restricting the usage of antigenspecific T-cell immunotherapy in a wide range of cancers.

Cancer/testis antigens (CTAs) are considered as ideal targets for cancer immunotherapy. They are expressed in a wide range of malignancies, while in normal tissues their expression is restricted in germline cells. ${ }^{8-10}$ Germ cells can escape from T-cell-induced attack due to the downregulation of HLA class I molecules. Thus, T cells can exclusively identify and attack cancer cells expressing corresponding CTA but spare normal somatic cells. ${ }^{11}$ Along with bladder cancer, melanoma, and ovarian cancer, non-small cell lung cancer (NSCLC) is found to have the highest frequency of CTA expression. ${ }^{11}$ Thus, CTA-specific T-cell immunotherapy may be more promising in NSCLC treatment.

So far, there are over 200 distinguished CTAs in CT database (http://www.cta.lncc.br), and 39\% (90/232) CTAs are demonstrated to be expressed in NSCLC. ${ }^{11,12}$ However, CTA expression has significant regional and racial difference. For example, MAGEA3 expression in NSCLC ranges from $35 \%$ to $60 \%$ in studies involving American and European patients. ${ }^{13,14}$ Previous studies of NSCLC mostly focused on the evaluation of a subset of CTA expression. ${ }^{8,15-19}$ In addition, it is costly to establish the antigen-specific T-cell therapy library expressing different CTA expressions. In order to improve the feasibility of antigen-specific T-cell therapy, the screening of CTAs to achieve the goal of the widest range of CTA expression coverage with the least CTAs combination is of great value.

Currently, no CTA expression profiles are established in People's Republic of China. In this study, we aimed to establish a CTA profiling in resected pathological stage III NSCLC through a comprehensive analysis of 10 CTAs' expression. Stratified analysis was also performed based on variable clinicopathological characteristics to evaluate the best CTA combination covering the widest range of selected NSCLC cases. The construction of CTA expression profile will provide more potential for further study of antigenspecific T-cell immunotherapy.

\section{Materials and methods}

\section{Patients and specimens}

This study used 200 resected pathological stage III lung cancer tissue specimens (100 squamous cell carcinoma [SCC], 100 adenocarcinomas [ADCs], 1:1) for immunohistochemistry. The tissues were surgically obtained at our hospital between January 2012 and December 2014. This study was approved by the Ethics Committee of Harbin Medical University Cancer Hospital. Prior to the study, written informed consent was obtained from each patient. The tumor stage was classified based on the 8th Edition Lung Cancer Stage Classification. ${ }^{20}$

\section{CTA selection}

CTAs are categorized into multiple family members. In order to select representative CTAs to establish CTAs profiling, we chose CTAs of variable family numbers as soon as possible including MAGE family, newly identified families, and some other family numbers (Table 1).

\section{Immunohistochemistry}

The paraffin-embedded samples were sliced into $5 \mu \mathrm{m}$ sections. Each slice was heated at $70^{\circ} \mathrm{C}$ overnight and then dewaxed using a xylene and ethanol gradient. Then, 3\% hydrogen peroxide was used to block the endogenous peroxidase activity, and the specimens were heated in $0.01 \mathrm{mmol} / \mathrm{L}$ citrate buffer ( $\mathrm{pH} \mathrm{6.0)}$ for $10 \mathrm{~min}$. The sections were then incubated with the primary antibodies overnight at $4^{\circ} \mathrm{C}$. The primary antibodies and dilutions used in this study are listed in Table 1. The specimens were washed and then incubated in a secondary antibody for $20 \mathrm{~min}$ at room temperature. The specimens were visualized with 3,3'-diaminobenzidine (ZSGB-BIO Inc., Beijing, People's Republic of China) and counterstained with hematoxylin solution.

\section{Immunohistochemistry scoring}

CTA expression was assessed by two pathologists. The immunopositivity of the CTAs was evaluated in a semiquantitative manner for the intensity ( $0=$ negative, $1=$ weak positive, $2=$ moderate positive, $3=$ strong positive) and the percentage of positive tumor cells $(0 \%-100 \%)$. All tumors with $>20 \%$ of the cells showing $2+$ or $3+$ staining intensity were considered positive. ${ }^{21}$

\section{Hierarchical analysis}

We employed unsupervised hierarchical clustering analysis to demonstrate deep pairwise relationships between genes. This clustering method seeks to build a hierarchy of clusters. 
Table I List of immunomarkers used for the immunohistochemical staining of non-small cell lung cancer

\begin{tabular}{llllll}
\hline Genes & CTA family & Clone & Dilution & Antigen retrieval & Source \\
\hline MAGEAI & MAGEA & Rabbit polyclone & $1: 25$ & Citrate buffer & SAB Signalway \\
MAGEA3 & MAGEA & Rabbit polyclone & $1: 75$ & Citrate buffer & SAB Signalway \\
MAGEAIO & MAGEA & Rabbit polyclone & $1: 50$ & Citrate buffer & SAB Signalway \\
MAGEB2 & MAGEB & Rabbit polyclone & $1: 30$ & Citrate buffer & SAB Signalway \\
MAGECI & MAGEC & Rabbit polyclone & $1: 20$ & Citrate buffer & SAB Signalway \\
XAGEI & XAGEI/GAGED & Goat polyclone & $6.67 \mu g / \mathrm{mL}$ & Citrate buffer & Abcam, Cambridge \\
KK-LC-I & Other & Rabbit polyclone & $1: 50$ & Citrate buffer & SAB Signalway \\
CTAGIA/B & NY-ESO-I & Rabbit polyclone & $1: 25$ & Citrate buffer & SAB Signalway \\
$V C X I$ & VCX & Rabbit polyclone & $1: 50$ & Citrate buffer & Invitrogen, Carlsbad \\
VCX3A & VCX & Mouse polyclone & $7.2 \mu g / \mathrm{mL}$ & Citrate buffer & Sigma-Aldrich \\
\hline
\end{tabular}

Normalized Hamming distance was used as a metric, and clusters were formed by using farthest point algorithm. Result of clustering is shown as heatmap.

\section{Frequency of CTA expression analysis}

In order to estimate percent of patients who have specific genes, we wrote an algorithm in python programming language. The algorithm generated all possible permutations (without repetitions) of specific genes and estimated how many patients had this combination of genes. Such analysis was not only performed on all patients, but also on certain groups of patients as well. For instance, we did split patients according to their sex, histology, and smoking status, and performed the analysis.

\section{EGFR mutation examination}

Briefly, genomic DNA was extracted and purified from formalin-fixed paraffin-embedded NSCLC biopsies. The most common epidermal growth factor receptor (EGFR) mutations including exon 18, 19, 20, and 21 were assessed by using the PCR screening method. The assay was performed in 384-well reaction plates (Thermo Fisher Scientific, Waltham, MA, USA), and the reaction was carried out in $40 \mathrm{uL}$, containing $4 \mathrm{uL}$ of the TaqMan Master Mix (Thermo Fisher Scientific), primers and probes at a final concentration of $9 \mathrm{uM}$ and 2 $\mathrm{uM}$, respectively. The remaining volume was made up to 40 $\mathrm{uL}$ with PCR grade water. The reaction was carried out at $95^{\circ} \mathrm{C}$ for 5 minutes, followed by 46 cycles at $95^{\circ} \mathrm{C}$ for 40 seconds, $62^{\circ} \mathrm{C}$ for 20 seconds, and $60^{\circ} \mathrm{C}$ for 40 seconds, in the Thermo Fisher Scientific 7500 HT machine.

\section{Statistical analysis}

The overall survival (OS) time was defined from diagnosis to death or the last follow-up if the patients were still alive. Sufficient clinical data were available. The survival curves and $95 \%$ confidence intervals (CIs) were obtained using the Kaplan-Meier method, and the survival rate in different groups was compared by log-rank tests. The relationships between CTAs and clinicopathological features were assessed by the chi-squared test. Univariate analysis was used to evaluate the potential prognostic role of variables. The prognostic factors with $P$-values $<0.2$ in the univariate analysis were examined in the multivariate analysis. SPSS 16.0 statistical software was used for statistical analysis. All $P$-values $<0.05$ were considered statistically significant.

\section{Results \\ Patient characteristics}

The patient characteristics are listed in Table 2. Most patients were males $(66.0 \%, \mathrm{n}=132)$ and ever smokers $(61.5 \%$, $\mathrm{n}=123)$. The mean age for all cases was $56.61 \pm 8.45$ years (range 32-81 years). All patients enrolled were diagnosed at stage III: IIIA $(76.5 \%, \mathrm{n}=153)$ and IIIB $(23.5 \%, \mathrm{n}=47)$. The number of patients treated with postoperative adjuvant chemotherapy and thoracic radiotherapy was $104(52.0 \%)$ and 24 (12.0\%), respectively. The main pathological types were $\operatorname{ADC}(50.0 \%, \mathrm{n}=100)$ and $\mathrm{SCC}(50.0 \%, \mathrm{n}=100)$.

\section{CTA expression at protein level}

The negative and positive CTA expression profiles in NSCLC are listed in Figure 1. In 200 patients, CTA expression sequence from low to high was listed as MAGEA3, VCX1, VCX3A, MAGEB2, CTAG1A/B, XAGE1, MAGEC1, KK-LC-1, MAGEA10, and MAGEA1, range 25.5\%-60.0\% (Figure 2A). CTA expression in ADC and SCC patients ranged from $24.0 \%$ to $64.0 \%$ and from $17.0 \%$ to $56.0 \%$, respectively. MAGEA1 showed the highest frequency of CTA expression (Table 3).

\section{CTA co-expression and cluster analysis}

The majority of CTAs were co-expressed in our study; only $15.0 \%(n=30)$ of NSCLC cases did not express any CTA 
Table 2 Prognostic analysis of 10 CTAs at protein level

\begin{tabular}{|c|c|c|c|c|c|c|}
\hline \multirow[t]{2}{*}{ Variables } & \multicolumn{3}{|c|}{ Univariate analysis } & \multicolumn{3}{|c|}{ Multivariate analysis } \\
\hline & $\mathbf{O} / \mathbf{N}^{*}$ & $\%$ & $P$ & $95 \% \mathrm{Cl}$ & HR & $P$ \\
\hline Sex & & & 0.256 & & & \\
\hline Male & $59 / 132$ & 44.7 & & & & \\
\hline Female & $25 / 68$ & 36.8 & & & & \\
\hline Age (years) & & & 0.052 & $0.943-2.689$ & 1.593 & 0.081 \\
\hline$<65$ & $64 / 163$ & 39.3 & & & & \\
\hline$\geq 65$ & $20 / 37$ & 54.1 & & & & \\
\hline Smoking history & & & 0.744 & & & \\
\hline No & $32 / 77$ & 41.6 & & & & \\
\hline Yes & $52 / 123$ & 42.3 & & & & \\
\hline Pathological types & & & 0.966 & & & \\
\hline $\mathrm{ADC}$ & $42 / 100$ & 42.0 & & & & \\
\hline SCC & $42 / 100$ & 42.0 & & & & \\
\hline Chemotherapy & & & 0.020 & $0.454-1.120$ & 0.713 & 0.142 \\
\hline No & $48 / 96$ & 50.0 & & & & \\
\hline Yes & $36 / 104$ & 34.6 & & & & \\
\hline Thoracic radiotherapy & & & 0.161 & $0.254-1.381$ & 0.592 & 0.225 \\
\hline No & $78 / 176$ & 44.3 & & & & \\
\hline Yes & $6 / 24$ & 25.0 & & & & \\
\hline TNM stage & & & 0.034 & $1.033-2.767$ & 1.691 & 0.037 \\
\hline IIIA & $60 / 153$ & 39.2 & & & & \\
\hline IIIB & $24 / 47$ & 51.1 & & & & \\
\hline MAGEAI & & & 0.421 & & & \\
\hline Negative & $29 / 80$ & 36.3 & & & & \\
\hline Positive & $55 / 120$ & 45.8 & & & & \\
\hline MAGEA3 & & & 0.940 & & & \\
\hline Negative & $62 / 149$ & 41.6 & & & & \\
\hline Positive & $22 / 51$ & 43.1 & & & & \\
\hline MAGEAIO & & & 0.193 & $0.545-1.350$ & 0.858 & 0.508 \\
\hline Negative & $46 / 100$ & 46.0 & & & & \\
\hline Positive & $38 / 100$ & 38.0 & & & & \\
\hline MAGEB2 & & & 0.379 & & & \\
\hline Negative & $59 /|3|$ & 45.0 & & & & \\
\hline Positive & $25 / 69$ & 36.2 & & & & \\
\hline MAGECI & & & 0.319 & & & \\
\hline Negative & $46 / 107$ & 43.0 & & & & \\
\hline Positive & $38 / 93$ & 40.9 & & & & \\
\hline XAGEI & & & 0.137 & $0.436-1.170$ & 0.714 & 0.181 \\
\hline Negative & $59 / 127$ & 46.5 & & & & \\
\hline Positive & $25 / 73$ & 34.3 & & & & \\
\hline KK-LC-I & & & 0.289 & & & \\
\hline Negative & $39 / 105$ & 37.1 & & & & \\
\hline Positive & $45 / 95$ & 47.4 & & & & \\
\hline CTAGIA/B & & & 0.476 & & & \\
\hline Negative & $52 / 130$ & 40.0 & & & & \\
\hline Positive & $32 / 70$ & 45.7 & & & & \\
\hline VCXI & & & 0.494 & & & \\
\hline Negative & $59 / 147$ & 40.1 & & & & \\
\hline Positive & $25 / 53$ & 47.2 & & & & \\
\hline VCX3A & & & 0.846 & & & \\
\hline Negative & $63 / 146$ & 43.2 & & & & \\
\hline Positive & $21 / 54$ & 38.9 & & & & \\
\hline
\end{tabular}

Note: *Observed death number/total patient number in each group. The bold numbers represent that the $P$-value of the prognostic factors in univariate analysis is $<0.05$, which is significant in univariate analysis.

Abbreviations: CTAs, cancer/testis antigens; $\mathrm{Cl}$, confidence interval; HR, hazard ratio; ADC, adenocarcinoma; SCC, squamous cell carcinoma.

(Figure $2 \mathrm{C}$ and D). The frequencies of at least $1-5$ expressed CTAs were $85.0 \%, 74.5 \%, 65.0 \%, 52.5 \%$, and $43.5 \%$ of the total 200 cases, respectively. We also found the rates were
$87.0 \%, 80.0 \%, 71.0 \%, 60.0 \%$, and $53.0 \%$ for ADC patients. The results were $83.0 \%, 69.0 \%, 59.0 \%, 45.0 \%$, and $34.0 \%$ for SCC patients (Figure 2B). 


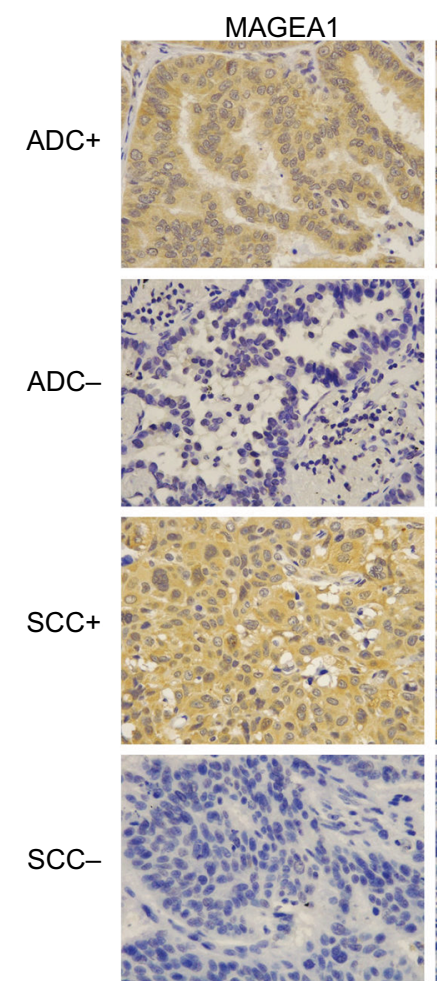

$$
\text { KK-LC-1 }
$$

$\mathrm{ADC}+$

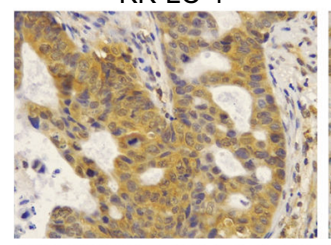

ADC-

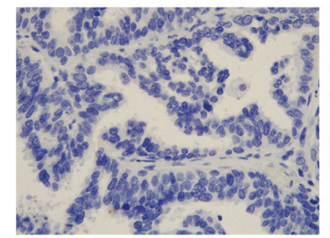

$\mathrm{SCC}+$

SCC-
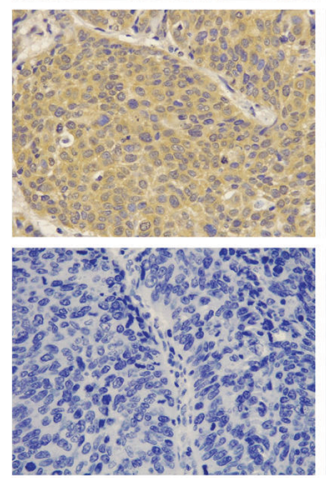
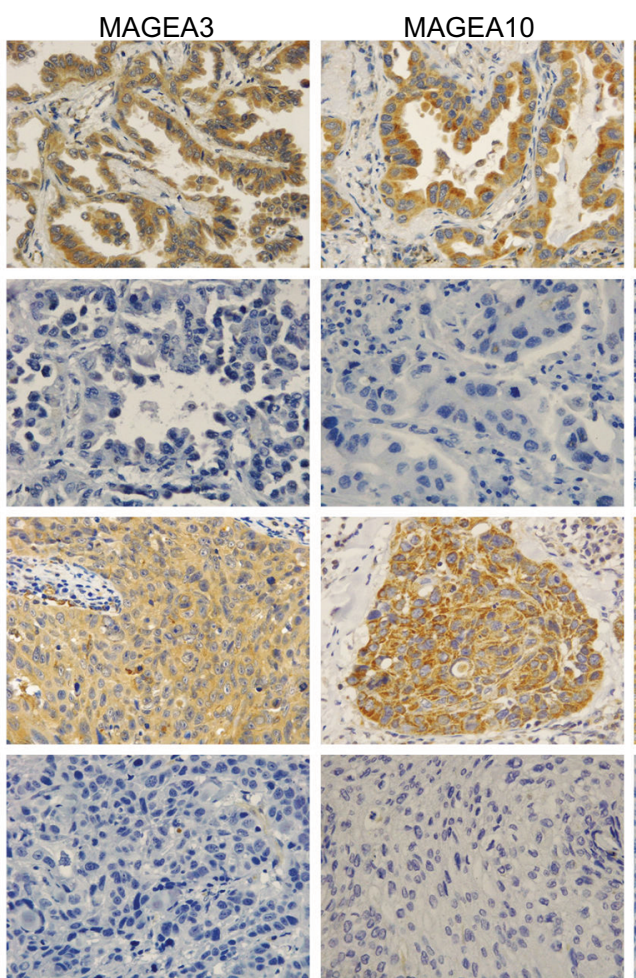

XAGE1
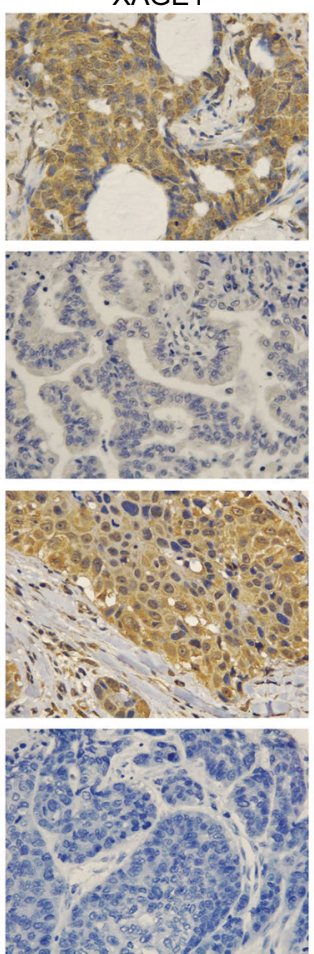
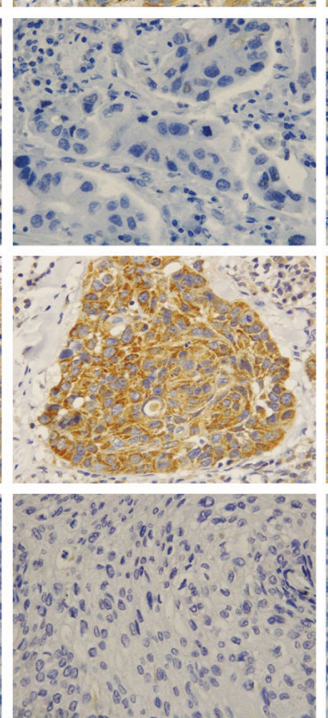

VCX1
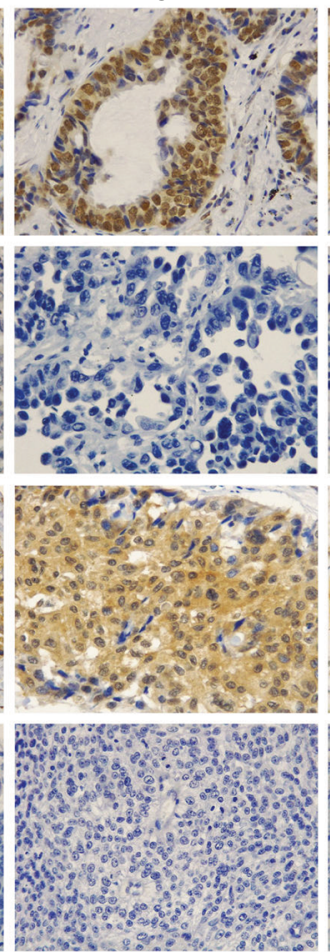
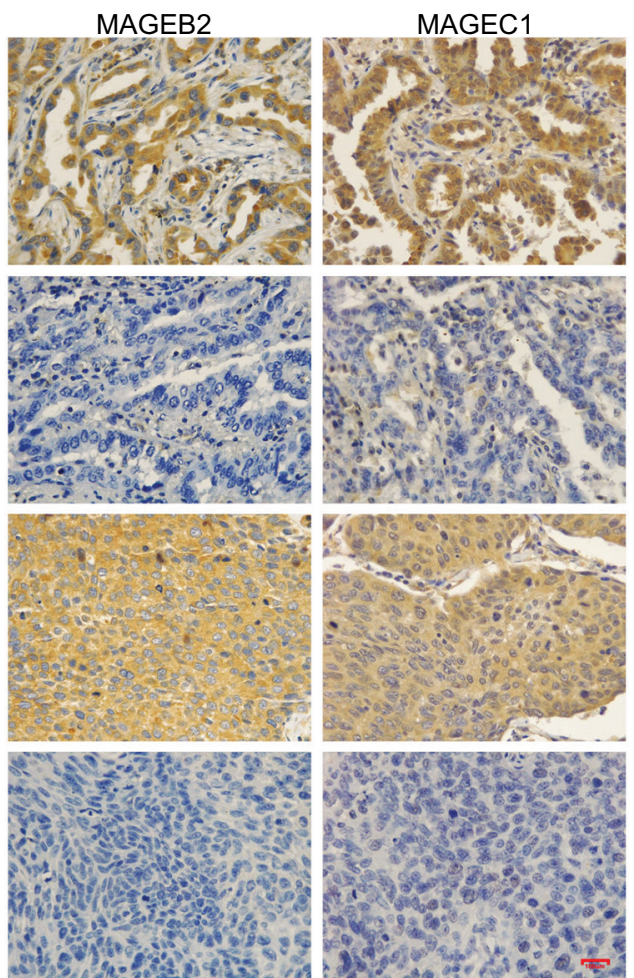

VCX3A
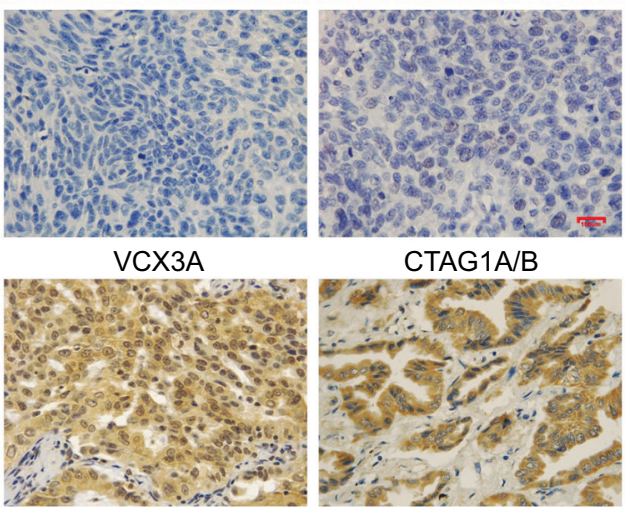

CTAG1A/B
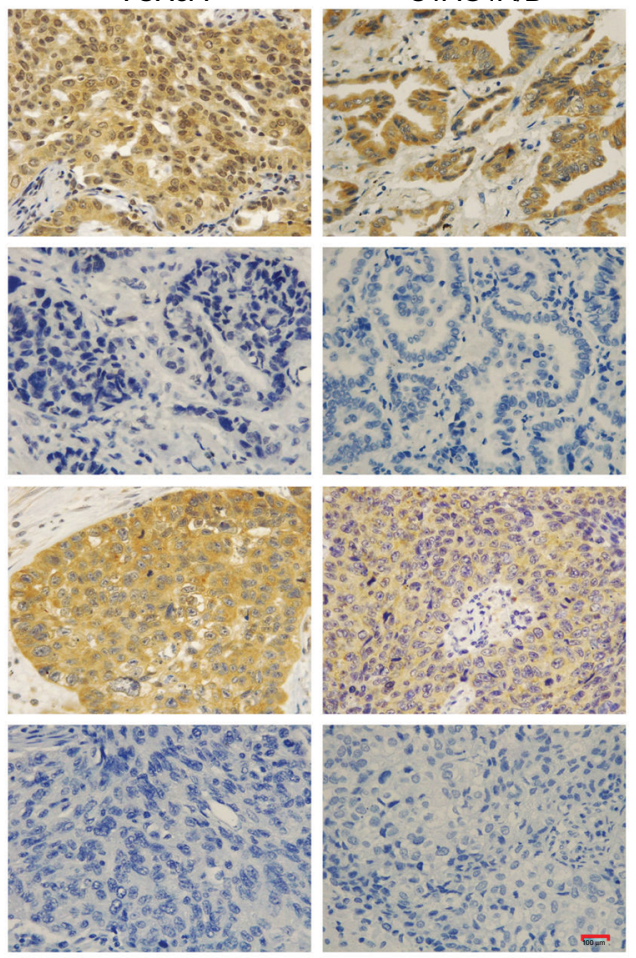

Figure I Positive and negative immunohistochemical staining of 10 selected CTAs in NSCLC according to pathological types.

Note: +, positive expression; -, negative expression.

Abbreviations: CTAs, cancer/testis antigens; NSCLC, non-small cell lung cancer; ADC, adenocarcinoma; SCC, squamous cell carcinoma.

Hierarchical clustering analysis was performed to assess CTA expression patterns among 200 NSCLC cases (Figure 3). The results showed that CTAs were co-expressed, one CTA usually expressed together with another one or several CTAs. For example, MAGEA1 expression was usually accompanied by MAGEC1 expression. In addition, 
A

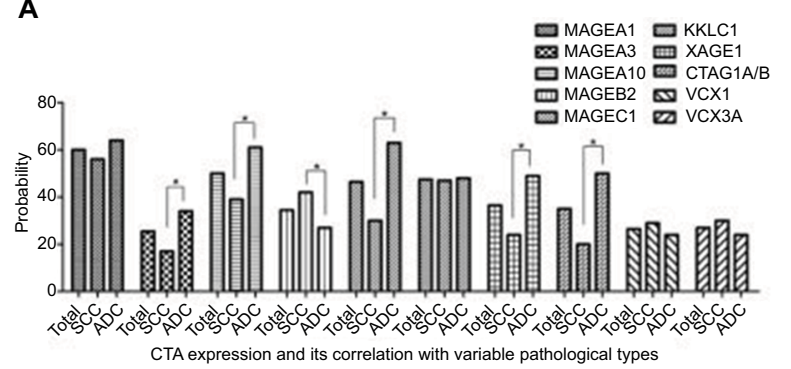

C

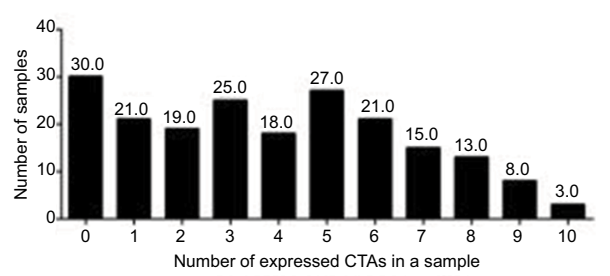

B

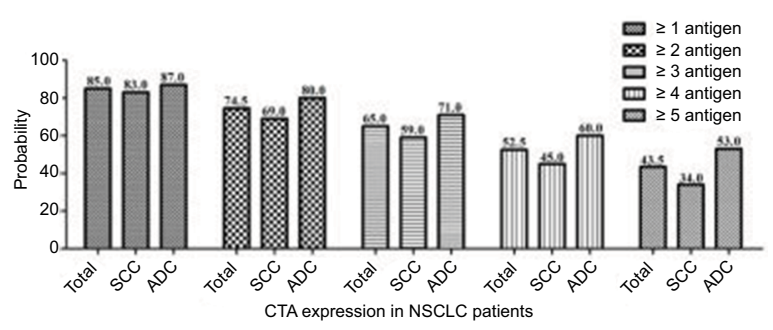

D

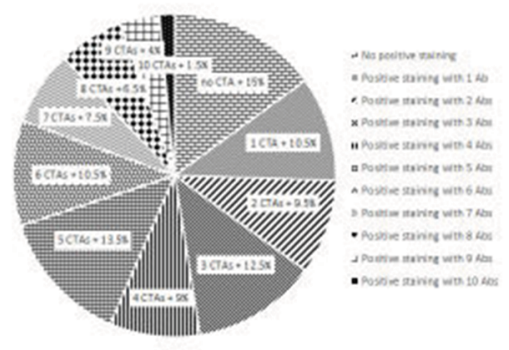

Figure 2 Expression of CTAs in NSCLC.

Notes: (A) CTA expression analysis in NSCLC. $* P<0.05$. (B) At least I-5 CTA positive expression in NSCLC. (C and D) CTA co-expression and proportion for all cases. Co-expression of the number of CTAs expressed in a NSCLC tumor sample is shown on the $x$-axis and the number of tumor samples is shown on the $y$-axis. Abbreviations: CTAs, cancer/testis antigens; NSCLC, non-small cell lung cancer; ADC, adenocarcinoma; SCC, squamous cell carcinoma.

CTA expression was found to be histology dependent. For example, XAGE1 showed a significant higher expression frequency in lung ADC.

\section{Relationship between CTA expression and clinicopathological features}

Of all CTAs, MAGEA3 (ADC vs SCC, 34.0\% vs 17.0\%, $P=0.006)$, MAGEA10 (61.0\% vs 39.0\%, $P=0.002)$, MAGEC1 $(63.0 \%$ vs $30.0 \%, P<0.001)$, XAGE1 $(49.0 \%$ vs $24.0 \%, P<0.001$, ) and CTAG1A/B (50.0\% vs $20.0 \%$, $P<0.001)$ had higher expression trends in ADC. MAGEB2 (ADC vs SCC, $27.0 \%$ vs $42.0 \%, P=0.026$ ) had an increased expression in SCC (Figure 2A, Table 3). We also found that MAGEC1 and XAGE1 were expressed more often in female patients. In addition, MAGEB2, MAGEC1, and XAGE1 expressions were closely associated with smoking history. No significant age and TNM differences were found between CTA expressions. CTA expression was closely correlated with sex, smoking history, and histology.

\section{Profiling CTAs in resected stage III lung cancer}

Any 2, 3, and 4 CTAs were coupled to explore the best CTA combinations that could cover the widest range of patients in our group. Of 10 CTAs, there were 45, 120 , and 210 conditions when any 2, 3, and 4 CTAs were combined. The best 2,3 , and 4 combinations were
MAGEA1+MAGEA10, MAGEA1+MAGEA10+CTAG1A/B, and MAGEA1+MAGEA10+CTAG1A/B+VCX1 (or MAG EA1+MAGEA10+MAGEB2+CTAG1A/B), which covered $72.0 \%, 76.5 \%$, and $79.5 \%$ of total cases, respectively. The top 10 combinations in 2, 3, and 4 CTAs groups are listed in Figure 4A.

\section{CTAs profiling based on clinicopathological characteristics}

Given that CTA expression was closely associated with sex, smoking history, and pathological types, stratified analysis was performed to assess the best CTA profiling for patients with variable clinicopathological features (Figures 4-6). Eight groups were categorized based on variable clinicopathological characteristics. For patients with features of male sex, positive smoking history, and $\mathrm{ADC}(\mathrm{n}=26,13.0 \%)$, the most commonly expressed CTA was MAGEA1 (80.8\%). Two CTA (MAGEA1+KK-LC-1) combination covered $92.3 \%$ of total cases, which was equal to 3 or 4 CTA combination (Figure $5 \mathrm{C})$. For patients with features of female sex, no smoking history, and ADC, 2, 3, and 4 CTA combination improved the coverage to $80.4 \%, 89.1 \%$, and $89.1 \%$ (Figure 4B), compared with $75.0 \%, 81.3 \%$, and $87.5 \%$ in patients with features of male sex, no smoking history, and ADC (Figure 5A). No significant coverage difference was found between 2 and 4 CTA combinations in female/positive smoking history/ADC and female/smoking history \pm /SCC patients (Figure $4 \mathrm{C}-\mathrm{E}$ ). 


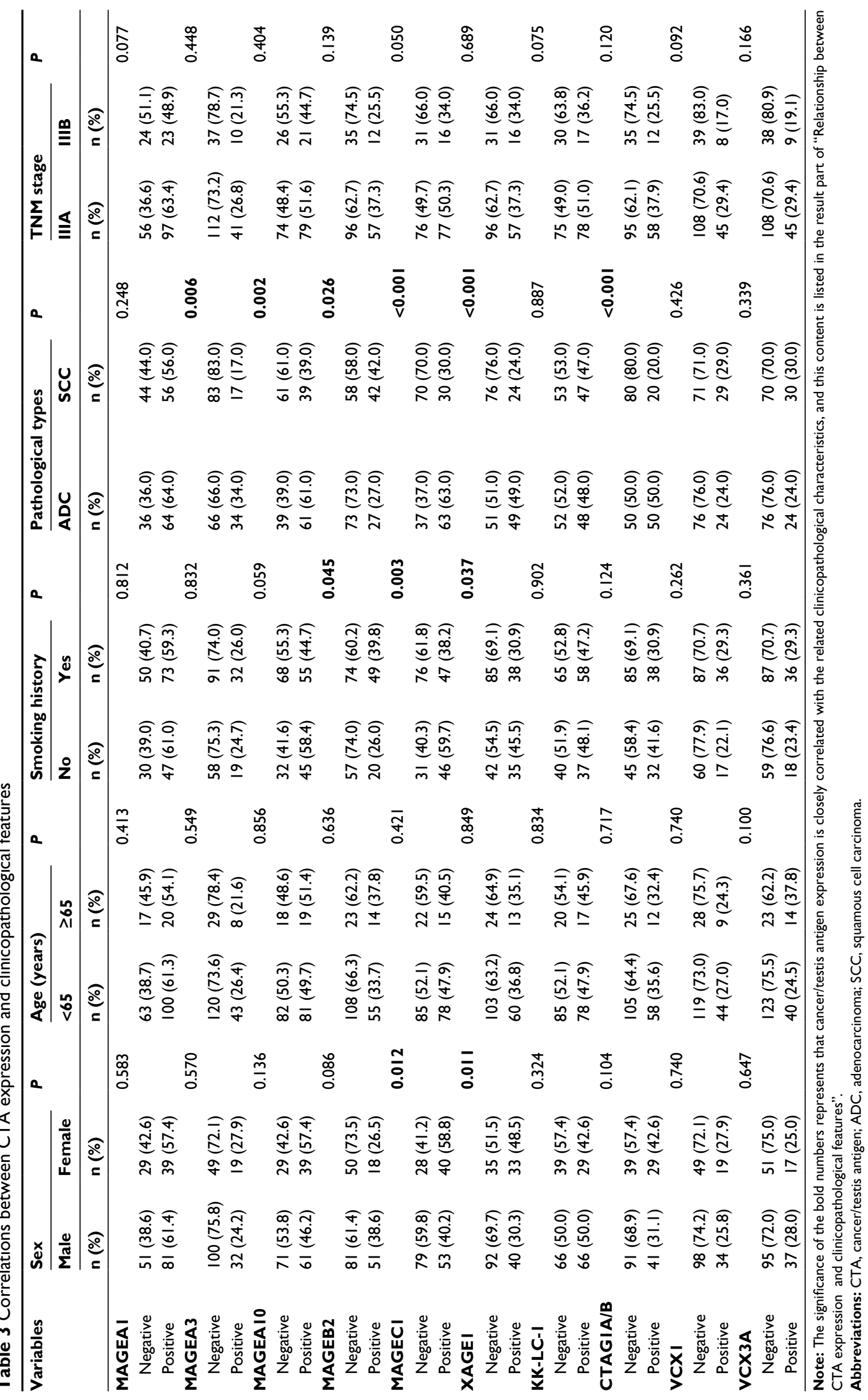




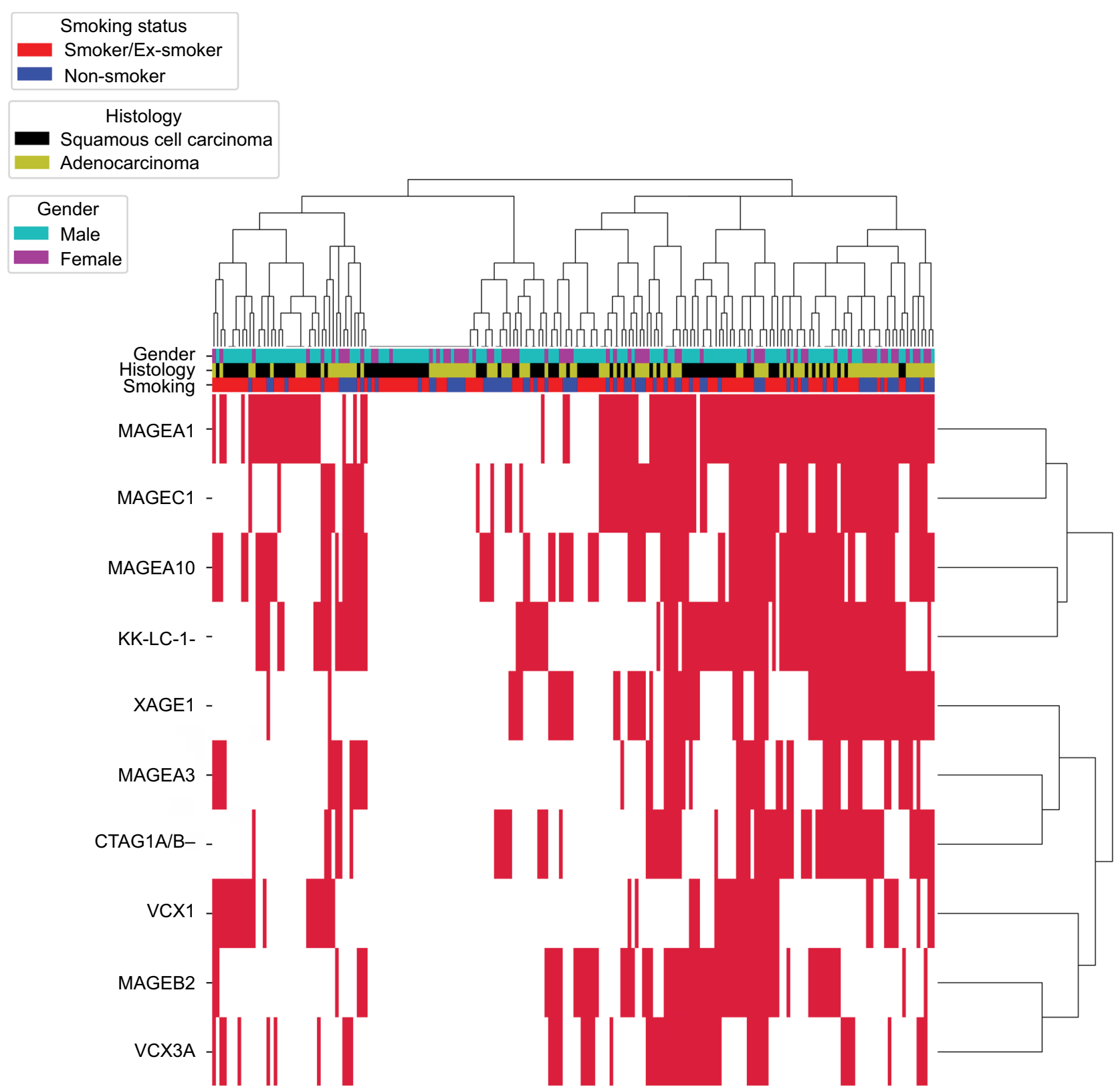

Figure 3 Hierarchical cluster analysis of CTAs in NSCLC

Notes: The 200 NSCLC cases were clustered based on the expression of the 10 CTAs in our group. The positive CTAs expression is marked red, and the negative expression is marked white.

Abbreviations: CTAs, cancer/testis antigens; NSCLC, non-small cell lung cancer.

Two CTA combination could best cover these patients. Even in the condition of 4 CTA combinations, the coverage was $\sim 80.0 \%$ for the other two groups (Figure $5 \mathrm{~B}$ and D). Detailed CTA combination information is given in Figure 6.

\section{CTA expression and OS}

There was no significant correlation between CTAs expression and OS for total cases $(P>0.05)$. In univariate analysis, postoperative adjuvant chemotherapy $(P=0.020)$ and TNM stage $(P=0.034)$ were found to be correlated with lung cancer prognosis. Only TNM stage (hazard ratio, $\mathrm{HR}=1.691,95 \%$ $\mathrm{CI}=1.033-2.767, P=0.037)$ held statistical significance in multivariate analysis (Table 2).

We also conducted subgroup analyses based on variable pathological types, and the results showed that adjuvant chemotherapy $(\mathrm{HR}=0.352,95 \% \mathrm{CI}=0.183-0.678, P=0.002)$ and VCX1 $(\mathrm{HR}=2.168,95 \% \mathrm{CI}=1.138-4.132, P=0.019)$ were independent prognostic factors for SCC patients (Figure 7A, Table 4). In ADC patients, we found that age ( $\mathrm{HR}=2.107,95 \%$ $\mathrm{CI}=1.010-4.398, P=0.047)$, TNM stage $(\mathrm{HR}=2.196,95 \%$ 

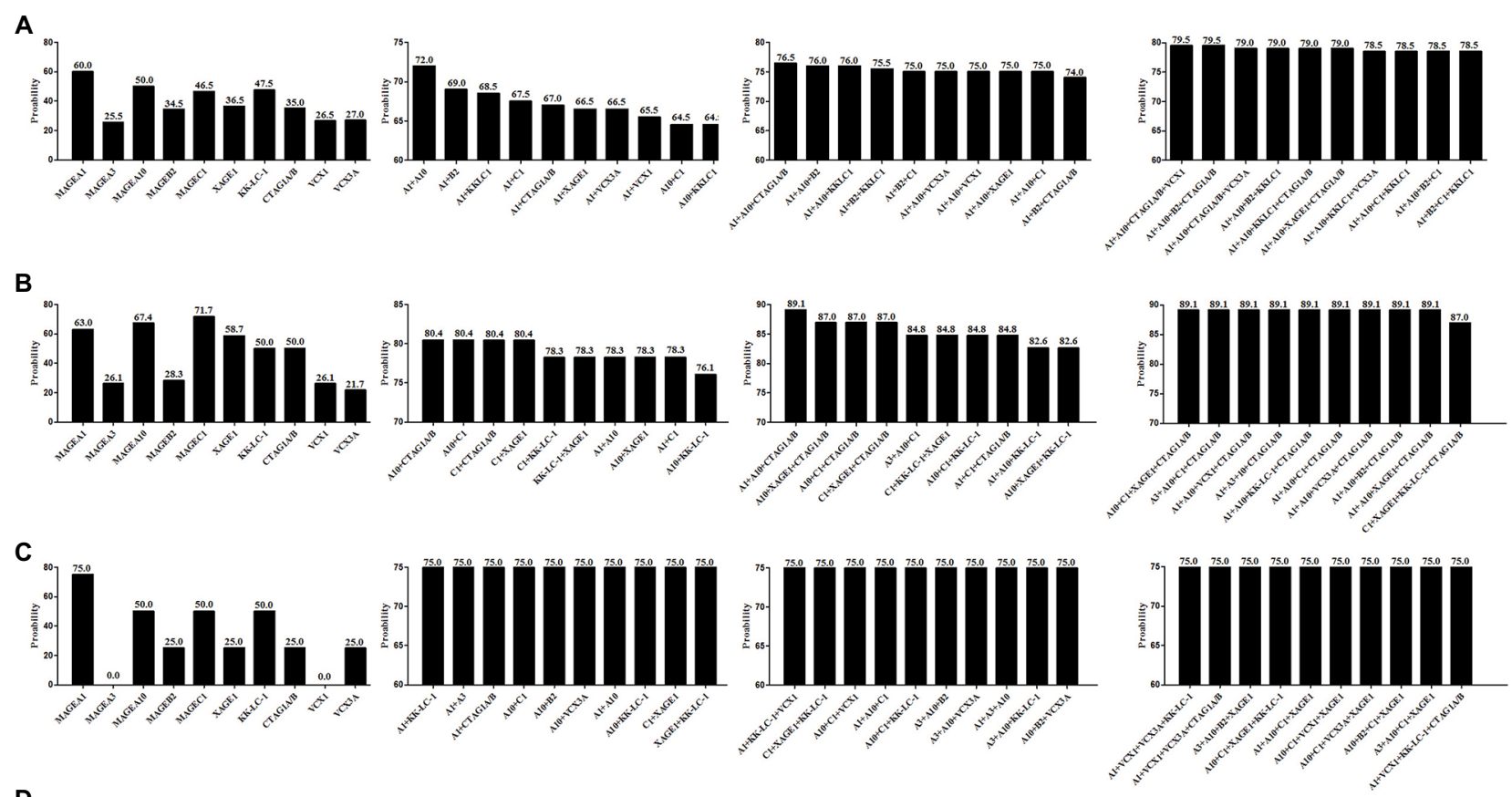

D
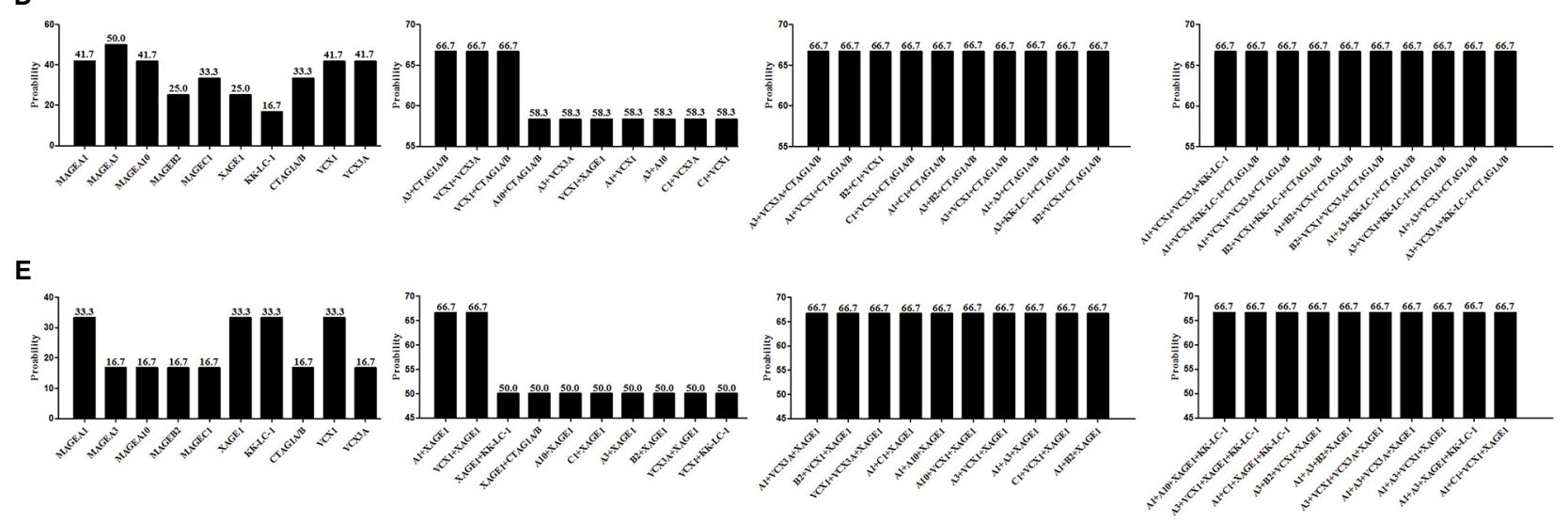

Figure 4 CTA profiling data based on any 2-4 CTA combination in total cases and precision CTA profiling data based on variable clinicopathological features.

Notes: (A) Single CTA expression and the best 2, 3, and 4 CTA combination covering the widest range of total 200 NSCLC cases. (B) CTA expression results for patients with the characteristics of female sex, no smoking history, and ADC. (C) CTA expression results for patients with the characteristics of female sex, no smoking history, and SCC. (D) CTA expression results for patients with the characteristics of female sex, positive smoking history, and ADC. (E) CTA expression results for patients with the characteristics of female sex, positive smoking history, and SCC.

Abbreviations: CTA, cancer/testis antigen; NSCLC, non-small cell lung cancer; ADC, adenocarcinoma; SCC, squamous cell carcinoma.

$\mathrm{CI}=1.094-4.408, P=0.027)$, and MAGEA10 $(\mathrm{HR}=0.530$, $95 \% \mathrm{CI}=0.287-0.979, P=0.043)$ were independent prognostic factors for these patients (Figure 7B). Age $<65$ years, stage IIIA, and positive MAGEA10 expression represented favorable prognosis.

Subgroup analysis based on patients treated with or without adjuvant chemotherapy showed that MAGEA3 $(\mathrm{HR}=2.056,95 \% \mathrm{CI}=1.055-4.008, P=0.034)$ and XAGE1 ( $\mathrm{HR}=0.502,95 \% \mathrm{CI}=0.260-0.968, P=0.040$ ) were independent prognostic factors in patients treated without adjuvant chemotherapy (Table 5). Patients with negative MAGEA3 and positive XAGE1 expression showed prolonged survival (Figure 7C and D). In addition, TNM stage ( $\mathrm{HR}=2.423,95 \%$
$\mathrm{CI}=1.160-5.063, P=0.019)$ was found to be an independent unfavorable prognostic factor in patients treated with postoperative adjuvant chemotherapy.

\section{Analysis of the OS of patients expressing XAGEI and MAGEA3}

Based on the subgroup analysis results, we aimed to assess the combined prognostic roles of XAGE1 and MAGEA3 in patients treated without adjuvant chemotherapy. Patients with XAGE1-positive expression and MAGEA3-negative expression showed prolonged OS (Figure 7E, $P=0.048$ ). We clustered these patients based on XAGE1 and MAGEA3 expression: Cluster A was expected to have a poorer 

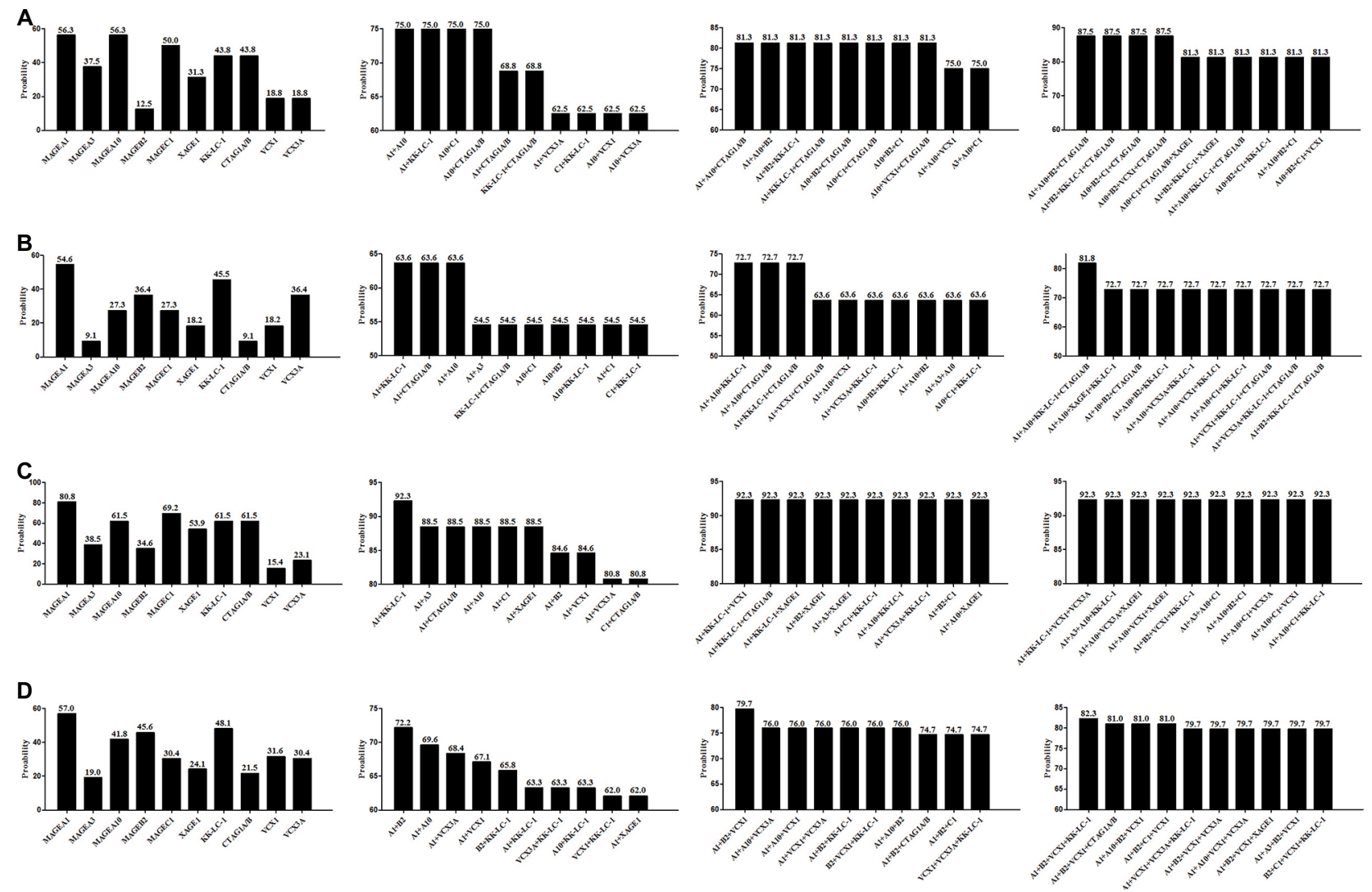

Figure 5 Precision CTA profiling data based on variable clinicopathological features.

Notes: (A) Single CTA expression and the best 2, 3, and 4 CTA combination for patients with the characteristics of male sex, no smoking history, and ADC. (B) CTA expression results for patients with the characteristics of male sex, no smoking history, and SCC. (C) CTA expression results for patients with the characteristics of male sex, positive smoking history, and ADC. (D) CTA expression results for patients with the characteristics of male sex, positive smoking history, and SCC.

Abbreviations: CTA, cancer/testis antigen; ADC, adenocarcinoma; SCC, squamous cell carcinoma.
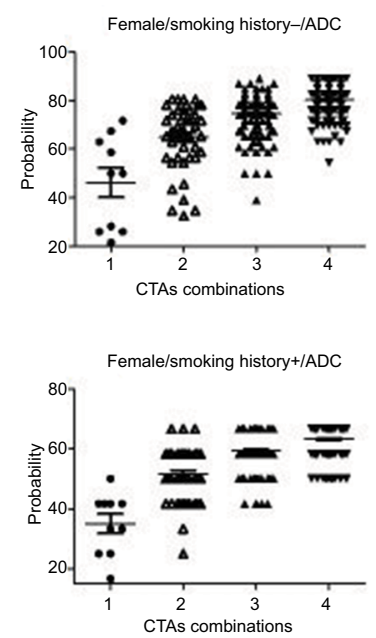
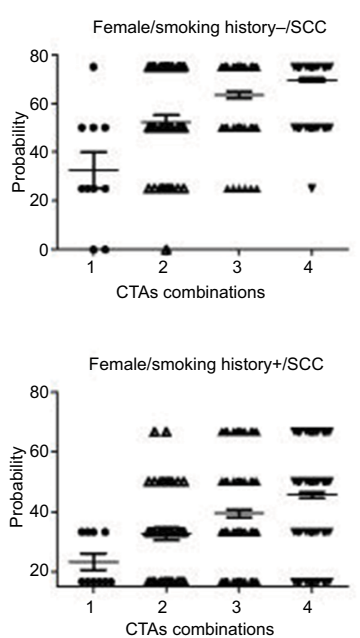
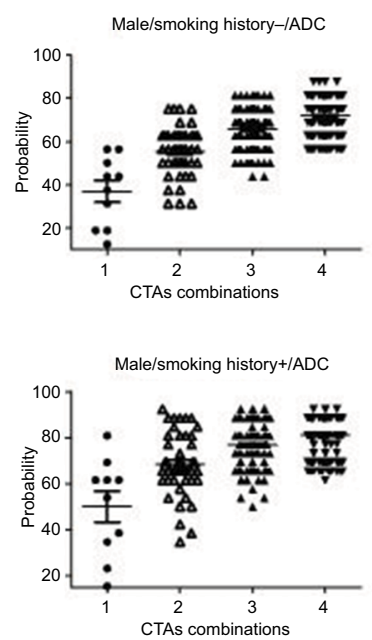

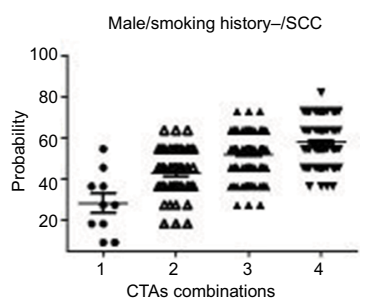

Male/smoking history+/SCC

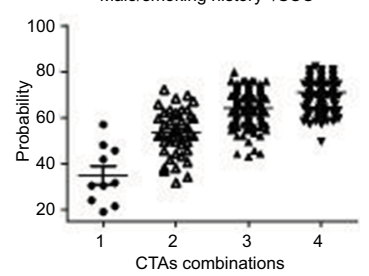

Figure 6 Establishment of CTA profiles to cover the widest range of NSCLC patients in our group based on variable sex, smoking history, and histology. Note: Each triangle represents one CTA combination.

Abbreviations: CTA, cancer/testis antigen; NSCLC, non-small cell lung cancer; ADC, adenocarcinoma; SCC, squamous cell carcinoma.

prognosis and included XAGE1-positive and MAGEA3positive, XAGE1-negative with either MAGEA3-negative or positive cases (Figure 7F). Cluster B was expected to have a prolonged survival and included XAGE1-positive and MAGEA3-negative cases $(P=0.010)$. We also examined the roles of XAGE1 and MAGEA3 in all 200 patients, and the 
A

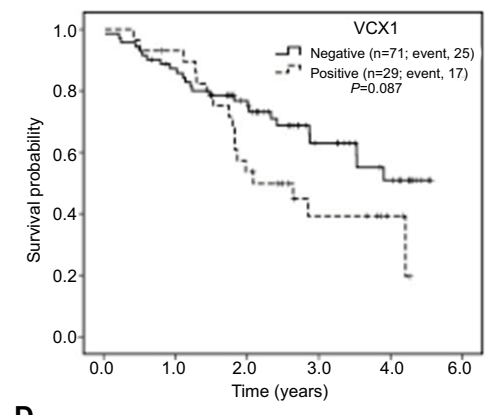

D

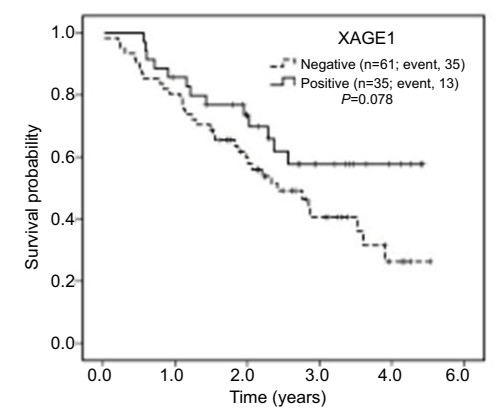

B

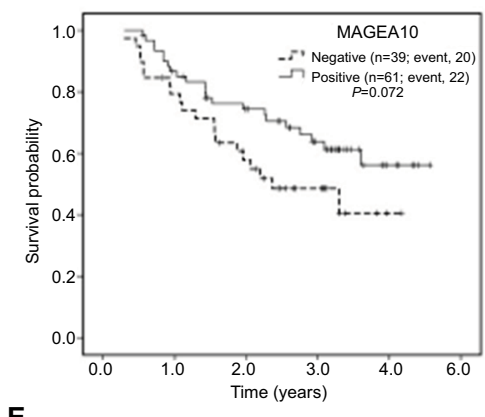

E

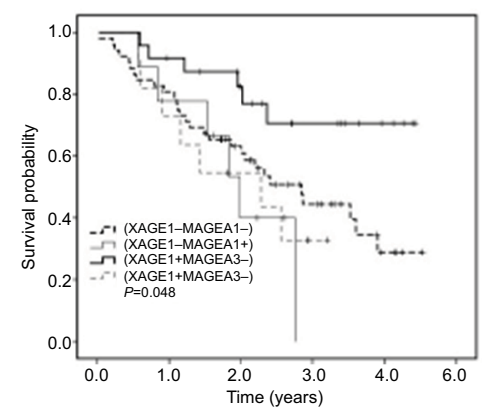

C

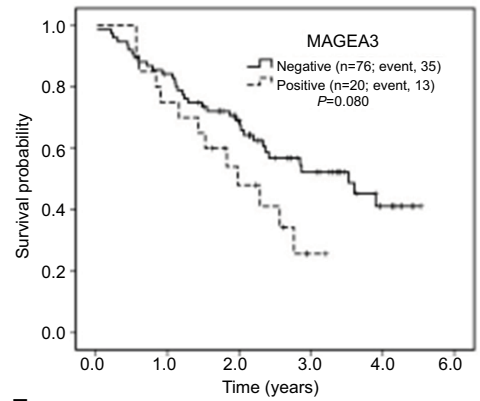

$\mathbf{F}$

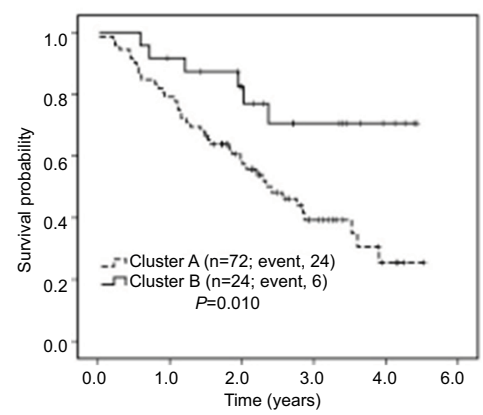

Figure 7 Prognostic roles of CTAs in non-small cell lung cancer.

Notes: (A) Positive VCXI expression was identified to be a negative prognostic factor of SCC patients based on subgroup analysis. (B) The favorable prognostic role of MAGEAIO in ADC patients. (C and D) Prognostic role of MAGEA3 and XAGEI in patients treated without adjuvant chemotherapy. (E) OS of patients stratified by the expression of MAGEA3 and XAGEI is shown by Kaplan-Meier method $(P=0.048)$. ( $F)$ Clusters $A$ and $B$ are classified in the text. Cluster $B$ shows a prolonged survival compared with Cluster $A(P=0.010)$.

Abbreviations: CTA, cancer/testis antigen; SCC, squamous cell carcinoma; ADC, adenocarcinoma; OS, overall survival.

results showed a similar trend. However, the $P$-value was not statistically significant $(P=0.130)$.

\section{CTA expression and EGFR mutant status}

EGFR tyrosine kinase inhibitors were demonstrated to greatly improve the disease-free survival of resected stage II-IIIA EGFR-mutant lung cancer patients. In our study, we also evaluated the association between CTA expression and EGFR mutant status. Of all ADC patients, only $8(8.0 \%)$ cases had definitive EGFR mutant and the rest 92 (92.0\%) had no or unknown EGFR mutant status. Unfortunately, our results showed CTA expression had no significant correlations with EGFR mutant status $(P>0.05)$ : MAGEA1 (no or unknown mutant status vs mutant, $62.0 \%$ vs $87.5 \%, P=0.253$ ), MAGEA3 (33.7\% vs $37.5 \%$, $P=1.000$ ), MAGEA10 (62.0\% vs $50.0 \%, P=0.708$ ), MAGEB2 (26.1\% vs $37.5 \%, P=0.443)$, MAGEC1 $(61.9 \%$ vs $75.0 \%$, $P=0.707$ ), XAGE1 ( $47.8 \%$ vs $62.5 \%, P=0.483)$, KK-LC-1 (45.7\% vs $75.0 \%, P=0.149)$, CTAG1A/B $(50.0 \%$ vs $50.0 \%$, $P=1.000)$, VCX1 (23.9\% vs $25.0 \%, P=1.000)$, and VCX3A ( $26.1 \%$ vs $0.0 \%, P=0.193$ ). In addition, no significant difference was found between EGFR mutant status and age, sex, smoking history, and TNM stage $(P>0.05)$. The relationship between CTA expression and EGFR mutant status remained to be studied.

\section{Discussion}

In this study, we comprehensively mapped CTA landscape in resected pathological stage III NSCLC based on CTA expression at protein level. Stratified analysis based on variable clinicopathological characteristics achieved the coverage of $92.3 \%$ with only 2 CTA combination in patients with features of male sex, positive smoking history, and ADC, compared with a $85.0 \%$ coverage for total cases when 10 CTAs were assessed. A maximum of $\sim 90.0 \%$ coverage can also be reached by 3 or 4 CTA combination in patients with features of male/female sex, no smoking history, and ADC. The goal of the maximum CTA expression coverage with the least CTA combination was achieved. In addition, selected CTAs were identified to be associated with prognosis on subgroup analysis. All results are of great significance for further study of CTA-specific T-cell immunotherapy.

For some reason, the patients enrolled in our study are all at stage III. On one hand, stage III NSCLC represents a heterogeneous group ranging from apparently resectable tumors with minimal mediastinal involvement to unresectable, bulky or contralateral nodal involvement nodal disease..$^{22,23}$ It shows a worse prognosis with a 5-year survival of $15 \%$, compared with that of $76 \%$ and $46 \%$ in early stage I and II 
Table 4 Subgroup analysis of CTA expression in variable pathological types

\begin{tabular}{|c|c|c|c|c|c|c|c|c|c|c|c|c|}
\hline \multirow[t]{3}{*}{ Variables } & \multicolumn{6}{|l|}{ SCC } & \multicolumn{6}{|l|}{ ADC } \\
\hline & \multicolumn{3}{|c|}{ Univariate analysis } & \multicolumn{3}{|c|}{ Multivariate analysis } & \multicolumn{3}{|c|}{ Univariate analysis } & \multicolumn{3}{|c|}{ Multivariate analysis } \\
\hline & $\mathbf{O} / \mathbf{N}$ & $\%$ & $P$ & $95 \% \mathrm{Cl}$ & HR & $\boldsymbol{P}$ & $\mathbf{O} / \mathbf{N}$ & $\%$ & $P$ & $95 \% \mathrm{Cl}$ & HR & $P$ \\
\hline Sex & & & 0.447 & & & & & & 0.291 & & & \\
\hline Male & $39 / 90$ & 43.3 & & & & & $20 / 42$ & 47.6 & & & & \\
\hline Female & $3 / 10$ & 30.0 & & & & & $22 / 58$ & 37.9 & & & & \\
\hline Age (years) & & & 0.203 & & & & & & 0.144 & $1.010-4.398$ & 2.107 & 0.047 \\
\hline$<65$ & $32 / 81$ & 39.5 & & & & & $32 / 82$ & 39.0 & & & & \\
\hline$\geq 65$ & $10 / 19$ & 52.6 & & & & & $10 / 18$ & 55.6 & & & & \\
\hline Smoking history & & & 0.689 & & & & & & 0.898 & & & \\
\hline No & $6 / 15$ & 40.0 & & & & & $26 / 62$ & 41.9 & & & & \\
\hline Yes & $36 / 85$ & 42.4 & & & & & $16 / 38$ & 42.1 & & & & \\
\hline Chemotherapy & & & 0.008 & $0.183-0.678$ & 0.352 & 0.002 & & & 0.526 & & & \\
\hline No & $26 / 48$ & 54.2 & & & & & $22 / 48$ & 45.8 & & & & \\
\hline Yes & $16 / 52$ & 30.8 & & & & & $20 / 52$ & 38.5 & & & & \\
\hline Thoracic radiotherapy & & & 0.466 & & & & & & 0.188 & $0.076-1.349$ & 0.321 & 0.121 \\
\hline No & $38 / 88$ & 43.2 & & & & & $40 / 88$ & 45.5 & & & & \\
\hline Yes & $4 / 12$ & 33.3 & & & & & $2 / 12$ & 16.7 & & & & \\
\hline TNM stage & & & 0.226 & & & & & & 0.088 & $1.094-4.408$ & 2.196 & 0.027 \\
\hline IIIA & $30 / 75$ & 40.0 & & & & & $30 / 78$ & 38.5 & & & & \\
\hline IIIB & $12 / 25$ & 48.0 & & & & & $12 / 22$ & 54.6 & & & & \\
\hline MAGEAI & & & 0.421 & & & & & & 0.726 & & & \\
\hline Negative & $15 / 44$ & 34.1 & & & & & $14 / 36$ & 38.9 & & & & \\
\hline Positive & $27 / 56$ & 48.2 & & & & & $28 / 64$ & 43.8 & & & & \\
\hline MAGEA3 & & & 0.834 & & & & & & 0.834 & & & \\
\hline Negative & $34 / 87$ & 39.1 & & & & & $28 / 66$ & 42.4 & & & & \\
\hline Positive & $8 / 17$ & 47.1 & & & & & $14 / 34$ & 41.2 & & & & \\
\hline MAGEAIO & & & 0.916 & & & & & & 0.072 & $0.287-0.979$ & 0.530 & 0.043 \\
\hline Negative & $26 / 61$ & 42.6 & & & & & $20 / 39$ & 51.3 & & & & \\
\hline Positive & $16 / 39$ & 41.0 & & & & & $22 / 61$ & 36.1 & & & & \\
\hline MAGEB2 & & & 0.526 & & & & & & 0.497 & & & \\
\hline Negative & $26 / 58$ & 44.8 & & & & & $33 / 73$ & 45.2 & & & & \\
\hline Positive & $16 / 42$ & 38.1 & & & & & $9 / 27$ & 33.3 & & & & \\
\hline MAGECI & & & 0.484 & & & & & & 0.389 & & & \\
\hline Negative & $29 / 70$ & 41.4 & & & & & $17 / 37$ & 46.0 & & & & \\
\hline Positive & $13 / 30$ & 43.3 & & & & & $25 / 63$ & 39.7 & & & & \\
\hline XAGE I & & & 0.120 & $0.238-1.224$ & 0.539 & 0.140 & & & 0.464 & & & \\
\hline Negative & $35 / 76$ & 46.1 & & & & & $24 / 51$ & 47.1 & & & & \\
\hline Positive & $7 / 24$ & 29.2 & & & & & $18 / 49$ & 36.7 & & & & \\
\hline KK-LC-I & & & 0.263 & & & & & & 0.712 & & & \\
\hline Negative & $18 / 53$ & 34.0 & & & & & $21 / 52$ & 40.1 & & & & \\
\hline Positive & $24 / 47$ & 51.1 & & & & & $21 / 48$ & 43.8 & & & & \\
\hline CTAGIA/B & & & 0.482 & & & & & & 0.752 & & & \\
\hline Negative & $31 / 80$ & 38.8 & & & & & $21 / 50$ & 42.0 & & & & \\
\hline Positive & $11 / 20$ & 55.0 & & & & & $21 / 50$ & 42.0 & & & & \\
\hline VCXI & & & 0.087 & $1.138-4.132$ & 2.168 & 0.019 & & & 0.464 & & & \\
\hline Negative & $25 / 71$ & 35.2 & & & & & $34 / 76$ & 44.7 & & & & \\
\hline Positive & $17 / 29$ & 58.6 & & & & & $8 / 24$ & 33.3 & & & & \\
\hline VCX3A & & & 0.264 & & & & & & 0.324 & & & \\
\hline Negative & $32 / 70$ & 45.7 & & & & & $31 / 76$ & 40.8 & & & & \\
\hline Positive & $10 / 30$ & 33.3 & & & & & $\mathrm{II} / 24$ & 45.8 & & & & \\
\hline
\end{tabular}

Note: Only factors with a $P$-value $<0.05$ are labeled as bolded numbers.

Abbreviations: CTA, cancer/testis antigen; SCC, squamous cell carcinoma; ADC, adenocarcinoma; $\mathrm{Cl}$, confidence interval; $\mathrm{HR}$, hazard ratio; O/N, observed death number/ total patient number in each group.

patients. ${ }^{23}$ On the other hand, stage IV patients are usually enough surgical samples, we choose stage III patients for diagnosed by needle biopsy with limited tumor samples. our present study. Interestingly, both stage IIIA and IIIB Considering the poor prognosis as well as the assurance of patients were included in our study. Our study was a ret- 
Table 5 Subgroup analysis of CTA expression in patients treated with or without postoperative adjuvant chemotherapy

\begin{tabular}{|c|c|c|c|c|c|c|c|c|c|c|c|c|}
\hline \multirow[t]{3}{*}{ Variables } & \multicolumn{6}{|c|}{ Without Chemo- } & \multicolumn{6}{|c|}{ With Chemo- } \\
\hline & \multicolumn{3}{|c|}{ Univariate analysis } & \multicolumn{3}{|c|}{ Multivariate analysis } & \multicolumn{3}{|c|}{ Univariate analysis } & \multicolumn{3}{|c|}{ Multivariate analysis } \\
\hline & $\mathbf{O} / \mathbf{N}$ & $\%$ & $P$ & $95 \% \mathrm{Cl}$ & HR & $P$ & $\mathbf{O} / \mathbf{N}$ & $\%$ & $P$ & $95 \% \mathrm{Cl}$ & HR & $P$ \\
\hline Sex & & & 0.099 & $0.349-1.582$ & 0.743 & $0.44 I$ & & & 0.912 & & & \\
\hline Male & $36 / 65$ & 55.4 & & & & & $23 / 67$ & 34.3 & & & & \\
\hline Female & $|2 / 3|$ & 38.7 & & & & & $13 / 37$ & 35.1 & & & & \\
\hline Age (years) & & & 0.245 & & & & & & 0.378 & & & \\
\hline$<65$ & $33 / 70$ & 47.1 & & & & & $31 / 93$ & 33.3 & & & & \\
\hline$\geq 65$ & $15 / 26$ & 57.7 & & & & & $5 / 11$ & 45.5 & & & & \\
\hline Smoking history & & & 0.092 & $0.685-2.958$ & 1.423 & 0.344 & & & 0.174 & $0.32 I-1.189$ & 0.617 & 0.149 \\
\hline No & $14 / 33$ & 42.4 & & & & & $18 / 44$ & 40.9 & & & & \\
\hline Yes & $34 / 63$ & 54.0 & & & & & $18 / 60$ & 30.0 & & & & \\
\hline Thoracic radiotherapy & & & 0.183 & $0.045-2.374$ & 0.326 & 0.269 & & & 0.707 & & & \\
\hline No & $47 / 90$ & 52.2 & & & & & $31 / 86$ & 36.0 & & & & \\
\hline Yes & $1 / 6$ & 16.7 & & & & & $5 / 18$ & 27.8 & & & & \\
\hline Pathology & & & 0.320 & & & & & & 0.313 & & & \\
\hline$A D C$ & $22 / 48$ & 45.8 & & & & & $20 / 52$ & 38.5 & & & & \\
\hline SCC & $26 / 48$ & 54.2 & & & & & $16 / 52$ & 30.8 & & & & \\
\hline TNM stage & & & 0.565 & & & & & & 0.019 & I.160-5.063 & 2.423 & 0.019 \\
\hline IIIA & $34 / 70$ & 48.6 & & & & & $26 / 83$ & 31.3 & & & & \\
\hline IIIB & $14 / 26$ & 53.9 & & & & & $10 / 2 \mid$ & 47.6 & & & & \\
\hline MAGEAI & & & 0.980 & & & & & & 0.203 & & & \\
\hline Negative & $|9 / 4|$ & 46.3 & & & & & $10 / 39$ & 25.6 & & & & \\
\hline Positive & $29 / 55$ & 52.7 & & & & & $26 / 65$ & 40.0 & & & & \\
\hline MAGEA3 & & & 0.080 & I.055-4.008 & 2.056 & 0.034 & & & 0.312 & & & \\
\hline Negative & $35 / 76$ & 46.1 & & & & & $27 / 73$ & 37.0 & & & & \\
\hline Positive & $13 / 20$ & 65.0 & & & & & $9 / 31$ & 29.0 & & & & \\
\hline MAGEAIO & & & 0.473 & & & & & & 0.270 & & & \\
\hline Negative & $26 / 49$ & 53.1 & & & & & $20 / 51$ & 39.2 & & & & \\
\hline Positive & $22 / 47$ & 46.8 & & & & & $16 / 53$ & 30.2 & & & & \\
\hline MAGEB2 & & & 0.912 & & & & & & 0.379 & & & \\
\hline Negative & $34 / 66$ & 51.5 & & & & & $25 / 65$ & 38.5 & & & & \\
\hline Positive & $14 / 30$ & 46.7 & & & & & $11 / 39$ & 28.2 & & & & \\
\hline MAGECI & & & 0.283 & & & & & & 0.653 & & & \\
\hline Negative & $26 / 50$ & 52.0 & & & & & $20 / 57$ & 35.1 & & & & \\
\hline Positive & $22 / 46$ & 47.8 & & & & & $16 / 47$ & 34.0 & & & & \\
\hline XAGE I & & & 0.078 & $0.260-0.968$ & 0.502 & 0.040 & & & 0.753 & & & \\
\hline Negative & $35 / 61$ & 57.4 & & & & & $24 / 66$ & 36.4 & & & & \\
\hline Positive & $13 / 35$ & 37.1 & & & & & $12 / 38$ & 31.6 & & & & \\
\hline KK-LC-I & & & 0.408 & & & & & & 0.392 & & & \\
\hline Negative & $24 / 56$ & 42.9 & & & & & $15 / 49$ & 30.6 & & & & \\
\hline Positive & $24 / 40$ & 60.0 & & & & & $21 / 55$ & 38.2 & & & & \\
\hline CTAGIA/B & & & 0.687 & & & & & & 0.677 & & & \\
\hline Negative & $29 / 60$ & 48.3 & & & & & $23 / 70$ & 32.9 & & & & \\
\hline Positive & $19 / 36$ & 52.8 & & & & & $13 / 34$ & 38.2 & & & & \\
\hline VCXI & & & 0.343 & & & & & & 0.302 & & & \\
\hline Negative & $39 / 82$ & 47.6 & & & & & $20 / 65$ & 30.8 & & & & \\
\hline Positive & $9 / 14$ & 64.3 & & & & & $16 / 39$ & 41.0 & & & & \\
\hline VCX3A & & & 0.840 & & & & & & 0.946 & & & \\
\hline Negative & $38 / 75$ & 50.7 & & & & & $25 / 71$ & 35.2 & & & & \\
\hline Positive & $10 / 21$ & 47.6 & & & & & $11 / 33$ & 33.3 & & & & \\
\hline
\end{tabular}

Note: Only factors with a $P$-value $<0.05$ are labeled as bolded numbers.

Abbreviations: CTA, cancer/testis antigen; Chemo-, chemotherapy; $\mathrm{Cl}$, confidence interval; HR, hazard ratio; ADC, adenocarcinoma; SCC, squamous cell carcinoma; O/N, observed death number/total patient number in each group.

rospective study and patients were enrolled in 2012-2014, while we used the newest 8th Edition Lung Cancer Stage Classification to assess tumor stage, which resulted in a part of stage IIIA patients into IIIB. ${ }^{20}$ For example, patients with T
( $5 \mathrm{~cm}<\mathrm{T} \leq 7 \mathrm{~cm}$ or $\mathrm{T}>7 \mathrm{~cm}$ ) N2M0 belong to stage IIIA (T2/ T3N2M0) in the 7th edition stage classification, while they are classified as stage IIIB (T3/T4N2M0) in the 8th edition stage classification. ${ }^{20,24}$ 
Most CTAs are co-expressed, and a previous study involving 90 CTAs demonstrated that only $5.5 \%$ of NSCLC cases do not express any CTA. ${ }^{11}$ In our study, 10 CTA combination can induce a frequency of $15.0 \%$ NSCLC patients expressing no CTA. Single CTA expression results in a maximum coverage of $60.0 \%$ in total cases, while 2, 3, and 4 CTAs can improve the frequency to $72.0 \%, 76.5 \%$ and $79.5 \%$, respectively. In our group, CTA expression was closely associated with clinicopathological characteristics including histology, sex, and smoking history. Therefore, stratified analysis was performed to assess the best CTA profiling for patients with variable clinicopathological features. For patients with features of male sex, positive smoking history, and ADC, single MAGEA1 expression can cover $80.8 \%$ patients, while 2 CTA combination reaches the maximum coverage of $92.3 \%$. This is a significant improvement compared with a coverage rate of $85.0 \%$ for total patients assessed by 10 CTAs in our study. For patients with features of female sex, no smoking history, and ADC, the combination of 3 CTAs can cover the widest range of $89.1 \%$ cases. For patients with features of male sex, no smoking history, and ADC, a combination of 4 CTAs can reach a maximum coverage of $87.5 \%$ cases. With the help of computer programming language, the goal of the maximum CTA expression coverage was reached by using the least CTA combination in patients with variable clinicopathological features. In selected patients, the examination of 2 or 3 CTAs may be enough, which can cover $\sim 90.0 \%$ NSCLC cases. We can choose to examine different CTA combinations for patients with variable clinicopathological characteristics.

Our immunohistochemistry results reveal that half of CTAs have higher expression in ADC patients, while only MAGEB2 is highly expressed in SCC patients. In subgroup analysis, VCX1 is also demonstrated to be an independent negative prognostic factor in SCC patients. There are currently limited treatment options for SCC and the strategies remain unsatisfied. MAGEB2 and VCX1 may represent potential targets for SCC treatment. In addition, MAGEA10 is identified to be an independent favorable prognostic factor in ADC patients. These results provide a new direction in the further studies of lung cancer.

In subgroup analysis, MAGEA3 and XAGE1 are also demonstrated to be closely associated with OS. The negative role of MAGEA3 protein has been demonstrated in previous studies, and this is consistent with our results. ${ }^{10,25-29}$ MAGEA3 expression can significantly promote cell proliferation and tumor survival and suppress apoptosis, while silencing MAGEA3 can improve the chemotherapy sensitivity of tumor cells. ${ }^{30-32}$ The role of XAGE1 remains controversial at present. Previous study shows that the coexpression of XAGE1 antigen and MHC class I on tumor cells can contribute to a prolonged survival in NSCLC patients, while XAGE1 expression alone has no impact on OS. ${ }^{33}$ In other studies, XAGE1 antibody is found to be accompanied by XAGE1 antigen expression in half advanced ADC patients, leading to a better survival by eliciting CD4 and CD8 T-cell responses. ${ }^{18,34,35}$ In addition, patients can be grouped, based on the expression of MAGEA3 and XAGE1, into Cluster A, who had a poorer prognosis, and Cluster B, who had a prolonged survival.

In our study, no significant expression difference was found between stage IIIA and IIIB patients. In previous studies, it was found that CTA expression was closely correlated with tumor stage and advanced stage patients showed a higher CTAs expression. ${ }^{7,36}$ Only patients with stage III were enrolled in our study, while patients with early stage I-II were excluded. CTA expression difference may be more significant between patients with early stage I-II and locally advanced non-metastatic stage III. In addition, we are interested in whether CTAs expression has significant correlation with EGFR mutant status. Unfortunately, the result is negative. Due to the retrospective feature of our study and low EGFR mutant rate in SCC, only 8 lung ADC patients had definite EGFR mutant status results and we only evaluated the correlation between CTA expression and lung ADC. We will collect more samples to demonstrate their correlations in our further study.

In conclusion, CTAs are ideal candidates for CTA-specific T-cell immunotherapy. We have established a CTA profiling in resected pathological stage III NSCLC based on 10 CTAs. The goal of the maximum CTA expression coverage is reached by using the least CTA combination based on variable histology, sex, and smoking history. These results can provide more valuable evidences for further clinical usage of individualized CTA-specific T-cell immunotherapy.

\section{Acknowledgments}

We thank Huiying Li (Department of Pathology, Harbin Medical University Cancer Hospital, Harbin, People's Republic of China), Ke Pan, and Cassian Yee (Departments of Melanoma Medical Oncology and Immunology, MD Anderson Cancer Center, Houston, TX, USA) for their direction in this project. This work was supported by National Natural Scientific Foundation of China (No: 81673007, 81572824, 81672931 and 81773133) and Postdoctoral Scientific Research Development Fund of Heilongjiang Province (LBH-Q16155). 


\section{Disclosure}

The authors report no conflicts of interest in this work.

\section{References}

1. Chen W, Zheng R, Baade PD, et al. Cancer statistics in China, 2015. CA Cancer J Clin. 2016;66(2):115-132.

2. Hong QY, Wu GM, Qian GS, et al. Prevention and management of lung cancer in China. Cancer. 2015;121 Suppl 17:3080-3088.

3. Torre LA, Bray F, Siegel RL, Ferlay J, Lortet-Tieulent J, Jemal A. Global cancer statistics, 2012. CA Cancer J Clin. 2015;65(2):87-108.

4. Chapuis AG, Thompson JA, Margolin KA, et al. Transferred melanoma-specific CD8+ $\mathrm{T}$ cells persist, mediate tumor regression, and acquire central memory phenotype. Proc Natl Acad Sci U S A. 2012;109(12):4592-4597.

5. Yee C, Thompson JA, Byrd D, et al. Adoptive T cell therapy using antigenspecific $\mathrm{CD} 8+\mathrm{T}$ cell clones for the treatment of patients with metastatic melanoma: in vivo persistence, migration, and antitumor effect of transferred T cells. Proc Natl Acad Sci U S A. 2002;99(25):16168-16173.

6. Chapuis AG, Ragnarsson GB, Nguyen HN, et al. Transferred WT1reactive $\mathrm{CD} 8+\mathrm{T}$ cells can mediate antileukemic activity and persist in post-transplant patients. Sci Transl Med. 2013;5(174):174ra127.

7. Salmaninejad A, Zamani MR, Pourvahedi M, Golchehre Z, Hosseini Bereshneh A, Rezaei N. Cancer/testis antigens: expression, regulation, tumor invasion, and use in immunotherapy of cancers. Immunol Invest. 2016;45(7):619-640.

8. Nakagawa K, Noguchi Y, Uenaka A, et al. XAGE-1 expression in nonsmall cell lung cancer and antibody response in patients. Clin Cancer Res. 2005;11(5):5496-5503.

9. Curigliano G, Viale G, Ghioni M, et al. Cancer-testis antigen expression in triple-negative breast cancer. Ann Oncol. 2011;22(1):98-103.

10. Kim J, Reber HA, Hines OJ, et al. The clinical significance of MAGEA3 expression in pancreatic cancer. Int J Cancer. 2006;118(9): 2269-2275.

11. Djureinovic D, Hallstrom BM, Horie M, et al. Profiling cancer testis antigens in non-small-cell lung cancer. JCI Insight. 2016;1(10):e86837.

12. Almeida LG, Sakabe NJ, deOliveira AR, et al. CTdatabase: a knowledgebase of high-throughput and curated data on cancer-testis antigens. Nucleic Acids Res. 2009;37:D816-D819.

13. Melloni G, Ferreri AJ, Russo V, et al. Prognostic significance of cancertestis gene expression in resected non-small cell lung cancer patients. Oncol Rep. 2004;12(1):145-151.

14. Scanlan MJ, Altorki NK, Gure AO, et al. Expression of cancer-testis antigens in lung cancer: definition of bromodomain testis-specific gene (BRDT) as a new CT gene, CT9. Cancer Lett. 2000;150(2): 155-164.

15. Fukuyama $T$, Hanagiri $T$, Takenoyama $M$, et al. Identification of a new cancer/germline gene, KK-LC-1, encoding an antigen recognized by autologous CTL induced on human lung adenocarcinoma. Cancer Res. 2006;66(9):4922-4928.

16. Gjerstorff MF, Pøhl M, Olsen KE, Ditzel HJ. Analysis of GAGE, NY-ESO-1 and SP17 cancer/testis antigen expression in early stage non-small cell lung carcinoma. BMC Cancer. 2013;13:466.

17. Grah JJ, Katalinic D, Juretic A, Santek F, Samarzija M. Clinical significance of immunohistochemical expression of cancer/testis tumorassociated antigens (MAGE-A1, MAGE-A3/4, NY-ESO-1) in patients with non-small cell lung cancer. Tumori. 2014;100(1):60-68.

18. Ohue Y, Kurose K, Mizote Y, et al. Prolongation of overall survival in advanced lung adenocarcinoma patients with the XAGE1 (GAGED2a) antibody. Clin Cancer Res. 2014;20(19):5052-5063.

19. Ohue Y, Kurose K, Nozawa R, et al. Survival of lung adenocarcinoma patients predicted from expression of PD-L1, galectin-9, and XAGE1 (GAGED2a) on tumor cells and tumor-infiltrating T cells. Cancer Immunol Res. 2016;4(12):1049-1060.
20. Detterbeck FC, Boffa DJ, Kim AW, Tanoue LT. The Eighth Edition Lung Cancer Stage Classification. Chest. 2017;151(1):193-203.

21. Taguchi A, Taylor AD, Rodriguez J, et al. A search for novel cancer/ testis antigens in lung cancer identifies VCX/Y genes, expanding the repertoire of potential immunotherapeutic targets. Cancer Res. 2014;74(17):4694-4705.

22. Ramnath N, Dilling TJ, Harris LJ, et al. Treatment of stage III non-small cell lung cancer: diagnosis and management of lung cancer, 3rd ed: American College of Chest Physicians evidence-based clinical practice guidelines. Chest. 2013;143(Suppl 5):e314S-e340S.

23. Biswas T, Sharma N, Machtay M. Controversies in the management of stage III non-small-cell lung cancer. Expert Rev Anticancer Ther. 2014;14(3):333-347.

24. Goldstraw P, Crowley J, Chansky K, et al; International Association for the Study of Lung Cancer International Staging Committee; Participating Institutions. The IASLC lung cancer staging project: proposals for the revision of the TNM stage groupings in the forthcoming (seventh) edition of the TNM classification of malignant tumours. JThorac Oncol. 2007;2(8):706-714.

25. Laban S, Atanackovic D, Luetkens T, et al. Simultaneous cytoplasmic and nuclear protein expression of melanoma antigen-A family and NY-ESO-1 cancer-testis antigens represents an independent marker for poor survival in head and neck cancer. Int J Cancer. 2014;135(5): $1142-1152$.

26. Gure AO, Chua R, Williamson B, et al. Cancer-testis genes are coordinately expressed and are markers of poor outcome in non-small cell lung cancer. Clin Cancer Res. 2005;11(22):8055-8062.

27. Ayyoub M, Scarlata CM, Hamaï A, Pignon P, Valmori D. Expression of MAGE-A3/6 in primary breast cancer is associated with hormone receptor negative status, high histologic grade, and poor survival. J Immunother. 2014;37(2):73-76.

28. Olarte I, Martinez A, Ramos-Peñafiel C, et al. MAGE-A3 expression is an adverse prognostic factor in diffuse large B-cell lymphoma. Hematology. 2011;16(6):368-372.

29. Abikhair M, Roudiani N, Mitsui H, et al. MAGEA3 expression in cutaneous squamous cell carcinoma is associated with advanced tumor stage and poor prognosis. $J$ Invest Dermatol. 2017;137(3):775-778.

30. Jungbluth AA, Ely S, DiLiberto M, et al. The cancer-testis antigens CT7 (MAGE-C1) and MAGE-A3/6 are commonly expressed in multiple myeloma and correlate with plasma-cell proliferation. Blood. 2005;106(1):167-174.

31. Yang B, Wu J, Maddodi N, Ma Y, Setaluri V, Longley BJ. Epigenetic control of MAGE gene expression by the KIT tyrosine kinase. $J$ Invest Dermatol. 2007;127(9):2123-2128.

32. Atanackovic D, Hildebrandt $Y$, Jadczak A, et al. Cancer-testis antigens MAGE-C1/CT7 and MAGE-A3 promote the survival of multiple myeloma cells. Haematologica. 2010;95(5):785-793.

33. Kikuchi E, Yamazaki K, Nakayama E, et al. Prolonged survival of patients with lung adenocarcinoma expressing XAGE-1b and HLA class I antigens. Cancer Immun. 2008;8:13.

34. Ohue Y, Eikawa S, Okazaki N, et al. Spontaneous antibody, and CD4 and CD8 T-cell responses against XAGE-1b (GAGED2a) in non-small cell lung cancer patients. Int J Cancer. 2012;131(5):E649-E658.

35. Pandey JP, Namboodiri AM, Ohue Y, Oka M, Nakayama E. Genetic variants of immunoglobulin $\gamma$ and $\kappa$ chains influence humoral immunity to the cancer-testis antigen XAGE-1b (GAGED2a) in patients with non-small cell lung cancer. Clin Exp Immunol. 2014;176(1): 78-83.

36. Shigematsu $\mathrm{Y}$, Hanagiri T, Shiota $\mathrm{H}$, et al. Clinical significance of cancer/ testis antigens expression in patients with non-small cell lung cancer. Lung Cancer. 2010;68(1):105-110. 


\section{Publish your work in this journal}

Cancer Management and Research is an international, peer-reviewed open access journal focusing on cancer research and the optimal use of preventative and integrated treatment interventions to achieve improved outcomes, enhanced survival and quality of life for the cancer patient. The manuscript management system is completely online and includes a very quick and fair peer-review system, which is all easy to use. Visit http://www.dovepress.com/testimonials.php to read real quotes from published authors.

Submit your manuscript here: https://www.dovepress.com/cancer-management-and-research-journal 